
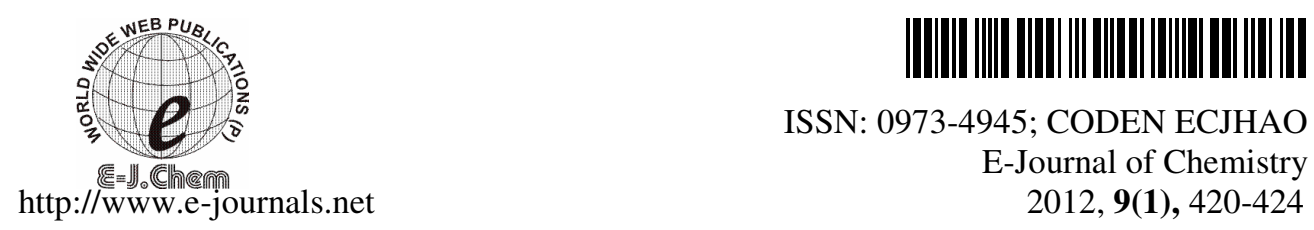

ISSN: 0973-4945; CODEN ECJHAO

E-Journal of Chemistry $2012,9(1), 420-424$

\title{
Synthesis of 3-(1, 3-Diphenyl-1H-pyrazol-4-yl) Propanoic Acids Using Diimide Reduction
}

\author{
M. DEEPA, V. HARINADHA BABU, \\ R. PARAMESHWAR and B. MADHAVA REDDY \\ Department of Pharmaceutical Chemistry \\ G. Pulla reddy College of Pharmacy, Mehdipatnam, Hyderabad-500028, India \\ parmi_pharma@yahoo.com \\ Received 2 July 2011; Accepted 27 August 2011

\begin{abstract}
Pyrazole-1H-4-yl-acrylic acids (3a-j) were prepared from pyrazole$1 H$-4-carbaldehydes which in turn were prepared by the Vilsmeier-Haack reaction of phenyl hydrazone derivatives (1a-j). The reaction of pyrazole- $1 H$ 4-yl-acrylic acids to 3-(1, 3-diphenyl-1 $H$-pyrazol-4-yl) propanoic acids (4a-j) was carried out using Pd-charcoal and diimide methods and \% yields were compared. Though the yields may be slightly less in diimide method, the method was found to be economical, highly effective with simple operating procedure.
\end{abstract}

Keywords: Pyrazole acrylic acids, Pyrazole propanoic acids, Diimide method

\section{Introduction}

In the last few years, considerable attention has been focused on pyrazole derivatives due to their interesting biological activities such analgesic ${ }^{1}$, antipyretic ${ }^{2}$, antiinflammatory ${ }^{3,4}$ antimicrobial $^{5}$, antiviral ${ }^{6,7}$, antidiabetic ${ }^{8}$, anticancer, ${ }^{9,10}$, estrogenic ${ }^{11}$ etc. In continuation of our work on pyrazole derivatives, in the present study, an attempt has been made to synthesize some novel pyrazole propanoic acid derivatives $(\mathbf{4 a - j})$ as per Scheme 1 with high purity and in reasonable yields. We predict that these pyrazole propanoic acids may be useful bioactive compounds in view of their individual biological nature.

\section{Experimental}

Melting points were determined in open capillary tubes using Analab melting point apparatus and are uncorrected. Purity of the compounds was verified by a single spot in TLC using E- Merck silica Gel $\mathrm{F}_{254}, 0.25 \mathrm{~mm}$ aluminum plates. Visualization was accomplished with UV light $(254 \mathrm{~nm})$ and iodine chamber. The IR spectra were recorded on Schimadzu FT-IR Spectrophotometer by using $1 \%$ potassium bromide discs. 


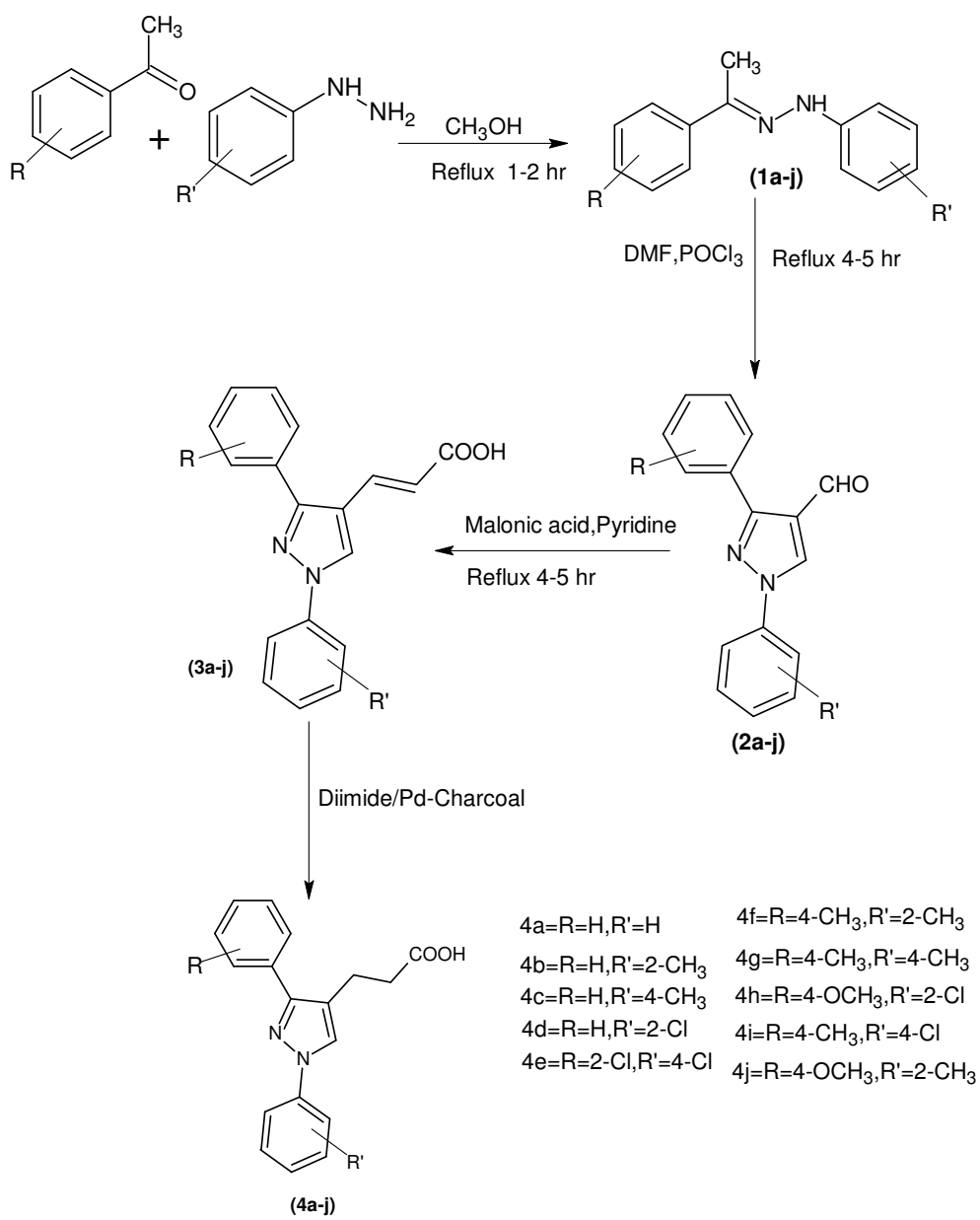

Scheme 1. Synthesis of pyrazole propanoic acid derivatives

Mass spectra of the compounds were recorded on mass spectrometer (Agilent 1100 series; EI/ESI-MS). All the ${ }^{1} \mathrm{H}$ NMR spectra were recorded on Brucker $300 \mathrm{MHz}$ Spectrometer using $\mathrm{CDCl}_{3} / \mathrm{DMSO}$ as solvent and tetramethylsilane as an internal Standard. Chemical shift values are listed in $\delta$ scale. Elemental analyses were carried out on a Carlo Erba 106 and Perkin Elmer model 240 analyzers.

\section{Results and Discussion}

Substituted phenyl hydrazones were prepared by heating substituted acetophenones with different hydrazines in methanol under reflux for $1-2 \mathrm{~h}$. Vilsmeier-Haack reaction ${ }^{12}$ of phenyl hydrazones using DMF and $\mathrm{POCl}_{3}$ afforded pyrazole-4-carbaldehydes in good yields and in high purity. The structures were confirmed on the basis of IR, ${ }^{1} \mathrm{H}$ NMR and mass spectral data. The aldehydes were converted into 3-(1, 3-diphenyl- $1 H$ - pyrazol-4-yl) acrylic acids (3a-j) by heating with malonic acid in pyridine and in the presence of catalytic amounts of piperidine in good yields (about 80\%). Usually, the reduction of double bond in $\alpha, \beta$-unsaturated acids is carried out using catalysts like Pd-charcoal, platinum, nickel, rhodium, nickel borohydride etc, which are highly expensive and in some cases disposal is 
the major problem. In the present study, the percentage yields of resulting 3-(1, 3-diphenyl1H-pyrazol-4-yl) propanoic acids (4a-j) obtained in both the methods are compared and the physical data including \% yields are reported in Table 1.

Table 1. Physical data of 3-(1, 3-diphenyl-1H-pyrazol-4-yl) propanoic acids (4a-j)

\begin{tabular}{cccccc}
\hline Compd & $\mathrm{R}$ & $\mathrm{R}^{1}$ & $\mathrm{~m} \cdot \mathrm{p}^{0} \mathrm{C}$ & \% Yield diimide method & \% Yield Pd-charcoal \\
\hline $\mathbf{4 a}$ & $\mathrm{H}$ & $\mathrm{H}$ & 100 & 68 & 79 \\
$\mathbf{4 b}$ & $\mathrm{H}$ & $2 \mathrm{CH}_{3}$ & 99 & 66 & 78 \\
$\mathbf{4 c}$ & $\mathrm{H}$ & $4 \mathrm{CH}_{3}$ & 98 & 65 & 77 \\
$\mathbf{4 d}$ & $\mathrm{H}$ & $2-\mathrm{C}$ & 98 & 67 & 72 \\
$\mathbf{4 e}$ & $2-\mathrm{CI}$ & $4-\mathrm{C}$ & 97 & 64 & 76 \\
$\mathbf{4 f}$ & $4-\mathrm{CH}_{3}$ & $2 \mathrm{CH}_{3}$ & 102 & 69 & 75 \\
$\mathbf{4 g}$ & $4-\mathrm{CH}_{3}$ & $4 \mathrm{CH}_{3}$ & 100 & 64 & 79 \\
$\mathbf{4 h}$ & $4-\mathrm{CH}_{3}$ & $2-\mathrm{C}$ & 98 & 65 & 78 \\
$\mathbf{4 i}$ & $4-\mathrm{CH}_{3}$ & $4-\mathrm{C}$ & 99 & 68 & 74 \\
$\mathbf{4 j}$ & $4-\mathrm{OCH}_{3}$ & $2 \mathrm{CH}_{3}$ & 101 & 66 & 75 \\
\hline
\end{tabular}

General procedure ${ }^{13}$

\section{1, 3-Diphenyl-1H-pyrazole-4-carbaldehyde (2a-j)}

To a mixture of DMF (0.1 mole) and phosphorous oxychloride (0.02 mole), ice-cold solution of phenyl hydrazone ( 0.01 mole) was added drop wise with stirring under ice-cold conditions. After the addition, the reaction mixture was refluxed and stirred at $60-70{ }^{\circ} \mathrm{C}$ for 4-5 h. Solution was cooled and poured into crushed ice and neutralized with $\mathrm{NaHCO}_{3}$ solution. The solid obtained was filtered under suction and recrystallised from methanol.

\section{3-(1,3-Diphenyl-1H-pyrazol-4yl) acrylic acid (3a-j)}

Pyrazole-4-carbaldehyde (0.01 mole) was dissolved in pyridine $(10 \mathrm{~mL})$ containing $(0.05$ mole $)$ malonic acid and a catalytic amount of piperidine $(0.5 \mathrm{~mL})$ and the reaction mixture was refluxed for $4-5 \mathrm{~h}$ on a water bath at $95-100{ }^{\circ} \mathrm{C}$. The resulting solution was poured into crushed ice with stirring and acidified with conc. $\mathrm{HCl}$ to remove any traces of pyridine. The resultant precipitate was filtered, washed with water and dilute $\mathrm{HCl}$, dried and recrystallized from glacial acetic acid or any appropriate solvent.

\section{3-(1, 3-Diphenyl-1H-pyrazole-4-yl) propanoic acid (4a-j): Diimide method}

3-(1,3-Diphenyl-1H-pyrazole-4-yl)acrylic acid (0.01 mole) was dissolved in hydrazine hydrate $(99 \%, 6 \mathrm{~mL})$ in a $250 \mathrm{~mL}$ conical flask and to the clear solution $20 \mathrm{~mL}$ of water was added. The flask was immersed in an ice bath and to which added few crystals of $\mathrm{CuSO}_{4}$ with stirring. To the cold solution, $10 \mathrm{~mL}$ of hydrogen peroxide $(30 \%)$ was added slowly such that the temperature remains below $30{ }^{\circ} \mathrm{C}$. After the addition was completed, the reaction mixture was allowed to stand in ice bath for $30 \mathrm{~min}$ followed by $10 \mathrm{~min}$ at room temperature. To the above mixture, a few $\mathrm{mL}$ of concentrated $\mathrm{HCl}$ was added with stirring. An oily product was separated which on cooling gave crystalline compound.

\section{Palladium-charcoal method}

To a solution of 3-(1, 3-diphenyl-1H-pyrazole-4-yl) acrylic acids (0.01 mole, $2.99 \mathrm{~g})$ in ethyl acetate $(30 \mathrm{~mL}), 20 \%$ palladium-charcoal $(0.1$ mole), ammonium formate $(0.2$ mole $)$ in ethyl acetate $(30 \mathrm{~mL})$ was added with stirring. The reaction mixture was stirred for overnight and the excess ethyl acetate was distilled off under reduced pressure. The precipitate was filtered, dried and recrystallized from appropriate solvent. 
4a:White solid: $\operatorname{IR}(\mathrm{KBr}) \mathrm{cm}^{-1}$ : $3438(\mathrm{O}-\mathrm{H}), 3013(\mathrm{C}-\mathrm{H}$ of aromatic ring $), 1708(\mathrm{C}=\mathrm{O}$ of $\mathrm{COOH}), 1596(\mathrm{C}=\mathrm{N}) ;{ }^{1} \mathrm{H} \mathrm{NMR}\left(\mathrm{CDCl}_{3}+\mathrm{DMSO}-\mathrm{d}_{6}\right): \delta 12.06\left(\mathrm{OH}, \mathrm{s}, 1 \mathrm{H}, \mathrm{D}_{2} \mathrm{O}\right.$ exchangeable $), 7.52-$ $7.59(\mathrm{Ar}-\mathrm{H}, \mathrm{m}, 11 \mathrm{H}), 2.71\left(\mathrm{CH}_{2} \mathrm{CH}_{2} \mathrm{COOH}, \mathrm{t}, 2 \mathrm{H}\right), 2.48\left(\mathrm{CH}_{2} \mathrm{CH}_{2} \mathrm{COOH}, \mathrm{t}, 2 \mathrm{H}\right)$; MS: $m / z .293$ $(\mathrm{M}+1)$; Anal.Calcd for $\mathrm{C}_{18} \mathrm{H}_{16} \mathrm{~N}_{2} \mathrm{O}_{2}$ : C,73.95; H, 5.52; N, 9.58. Found: C, 73.97; H, 5.49; N, 9.61\%.

4b: White solid: IR $(\mathrm{KBr}) \mathrm{cm}^{-1}: 3415(\mathrm{O}-\mathrm{H}), 3016(\mathrm{C}-\mathrm{H}$ of aromatic ring), $1712(\mathrm{C}=\mathrm{O}$ of $\mathrm{COOH}), 1628(\mathrm{C}=\mathrm{N}) ;{ }^{1} \mathrm{H} \mathrm{NMR}\left(\mathrm{CDCl}_{3}+\mathrm{DMSO}-\mathrm{d}_{6}\right): \delta 12.09\left(\mathrm{OH}, \mathrm{s}, 1 \mathrm{H}, \mathrm{D}_{2} \mathrm{O}\right.$ exchangeable $)$, 7.52-7.59( $\mathrm{Ar}-\mathrm{H}, \quad \mathrm{m}, 10 \mathrm{H}), \quad 3.14\left(\mathrm{CH}_{3}, \quad \mathrm{~s}, 3 \mathrm{H}\right), \quad 2.69\left(\mathrm{CH}_{2} \mathrm{CH}_{2} \mathrm{COOH}, \quad \mathrm{t}, \quad 2 \mathrm{H}\right), \quad 2.46$ $\left(\mathrm{CH}_{2} \mathrm{CH}_{2} \mathrm{COOH}, \mathrm{t}, 2 \mathrm{H}\right)$; MS: $\mathrm{m} / 2307(\mathrm{M}+1)$; Anal. Calcd for $\mathrm{C}_{19} \mathrm{H}_{18} \mathrm{~N}_{2} \mathrm{O}_{2}: \mathrm{C}, 74.49 ; \mathrm{H}$, 5.92; N, 9.14. Found: C, 74.51; H, 5.87; N, $9.16 \%$.

4c: White solid: IR (KBr)cm ${ }^{-1}: 3434(\mathrm{O}-\mathrm{H}), 3012\left(\mathrm{C}-\mathrm{H}\right.$ of aromatic ring), 2927(C-H of $\left.\mathrm{CH}_{3}\right)$, $1718(\mathrm{C}=\mathrm{O}$ of $\mathrm{COOH}), 1627(\mathrm{C}=\mathrm{N}) ;{ }^{1} \mathrm{H} \mathrm{NMR}\left(\mathrm{CDCl}_{3}+\mathrm{DMSO}_{6}\right): \delta 12.01\left(\mathrm{OH}, \mathrm{s}, 1 \mathrm{H}, \mathrm{D}_{2} \mathrm{O}\right.$ exchangeable), 7.52-7.59 (Ar-H, m, $10 \mathrm{H}), 3.14\left(\mathrm{CH}_{3}, \mathrm{~s}, 3 \mathrm{H}\right), 2.68\left(\mathrm{CH}_{2} \mathrm{CH}_{2} \mathrm{COOH}, \mathrm{t}, 2 \mathrm{H}\right)$, 2.42( $\left.\mathrm{CH}_{2} \mathrm{CH}_{2} \mathrm{COOH}, \mathrm{t}, 2 \mathrm{H}\right)$; MS: $\mathrm{m} / 2$ 307(M+1); Anal.Calcd for $\mathrm{C}_{19} \mathrm{H}_{18} \mathrm{~N}_{2} \mathrm{O}_{2}: \mathrm{C}, 74.49 ; \mathrm{H}$, 5.92; N, 9.14. Found :C, 74.52; H, 5.86; N, $9.18 \%$.

4d: Pale yellow solid: $\mathrm{IR}(\mathrm{KBr}) \mathrm{cm}^{-1}: 3438(\mathrm{O}-\mathrm{H}), 3011(\mathrm{C}-\mathrm{H}$ of aromatic ring $), 1706(\mathrm{C}=\mathrm{O}$ of $\mathrm{COOH}), 1612(\mathrm{C}=\mathrm{N}), 754(\mathrm{C}-\mathrm{Cl}) ;{ }^{1} \mathrm{H} \mathrm{NMR}\left(\mathrm{CDCl}_{3}+\mathrm{DMSO}_{-} \mathrm{d}_{6}\right): \delta 12.16\left(\mathrm{OH}, \mathrm{s}, 1 \mathrm{H}, \mathrm{D}_{2} \mathrm{O}\right.$ exchangeable) ,7.15-7.67(Ar-H,m,10H), 2.62( $\left.\mathrm{CH}_{2} \mathrm{CH}_{2} \mathrm{COOH}, \mathrm{t}, 2 \mathrm{H}\right), 2.44\left(\mathrm{CH}_{2} \mathrm{CH}_{2} \mathrm{COOH}, \mathrm{t}, 2 \mathrm{H}\right) ; \mathrm{MS}: \mathrm{m} / \mathrm{z}$ 327(M+1); Anal.Calcd for $\mathrm{C}_{18} \mathrm{H}_{15} \mathrm{ClN}_{2} \mathrm{O}_{2}$ : C, 66.16; H, 4.63; N, 8.57. Found : C, 66.19; H, 4.59; $\mathrm{N}, 8.60 \%$.

4e: Pale yellow solid: $\mathrm{IR}(\mathrm{KBr}) \mathrm{cm}^{-1}: 3408(\mathrm{O}-\mathrm{H}), 3013(\mathrm{C}-\mathrm{H}$ of aromatic ring ), $1708(\mathrm{C}=\mathrm{O}$ of $\mathrm{COOH})$, $1620(\mathrm{C}=\mathrm{N}), 756(\mathrm{C}-\mathrm{Cl}) ;{ }^{1} \mathrm{HNMR}\left(\mathrm{CDCl}_{3}+\mathrm{DMSO}-\mathrm{d}_{6}\right): \delta 12.18\left(\mathrm{OH}, \mathrm{s}, 1 \mathrm{H}, \mathrm{D}_{2} \mathrm{O}\right.$ exchangeable $), 7.15-$ 7.67(Ar-H, m, 9H), 2.58( $\left.\mathrm{CH}_{2} \mathrm{CH}_{2} \mathrm{COOH}, \mathrm{t}, 2 \mathrm{H}\right), 2.41\left(\mathrm{CH}_{2} \mathrm{CH}_{2} \mathrm{COOH}, \mathrm{t}, 2 \mathrm{H}\right) ; \mathrm{MS}: \mathrm{m} / z$ 362(M+1); Anal.Calcd for $\mathrm{C}_{18} \mathrm{H}_{14} \mathrm{Cl}_{2} \mathrm{~N}_{2} \mathrm{O}_{2}$ : C, 59.85; H, 3.91; N, 7.76. Found: C, 59.88; H, 3.88; N, 7.78.\%.

4f: White solid:IR(KBr $) \mathrm{cm}^{-1}$ : $3418(\mathrm{O}-\mathrm{H}), 3018(\mathrm{C}-\mathrm{H}$ of aromatic ring $), 1710(\mathrm{C}=\mathrm{O}$ of $\mathrm{COOH}), 1623(\mathrm{C}=\mathrm{N}) ;{ }^{1} \mathrm{HNMR}\left(\mathrm{CDCl}_{3}+\mathrm{DMSO}_{-} \mathrm{d}_{6}\right): \delta 12.09\left(\mathrm{OH}, \mathrm{s}, 1 \mathrm{H}_{2} \mathrm{D}_{2} \mathrm{O}\right.$ exchangeable $)$, 7.57-7.59(Ar-H, m, 9H), 3.24( $\left.\mathrm{CH}_{3}, \mathrm{~s}, 3 \mathrm{H}\right), 3.19\left(\mathrm{CH}_{3}, \mathrm{~s}, 3 \mathrm{H}\right), 2.71\left(\mathrm{CH}_{2} \mathrm{CH}_{2} \mathrm{COOH}, \mathrm{t}, 2 \mathrm{H}\right)$, 2.42 $\left(\mathrm{CH}_{2} \mathrm{CH}_{2} \mathrm{COOH}, \mathrm{t}, 2 \mathrm{H}\right)$; MS: $\mathrm{m} / z$ 321(M+1); Anal.Calcd for $\mathrm{C}_{20} \mathrm{H}_{20} \mathrm{~N}_{2} \mathrm{O}_{2}: \mathrm{C}, 74.98 ; \mathrm{H}$, 6. $29 ; \mathrm{N}, 8.74$. Found: C, 75.02; H, 6.25; N, $8.76 \%$.

4g: White solid: $\mathrm{IR}(\mathrm{KBr}) \mathrm{cm}^{-1}: 3405(\mathrm{O}-\mathrm{H}), 3016\left(\mathrm{C}-\mathrm{H}\right.$ of aromatic ring), 2857(C-H of $\left.\mathrm{CH}_{3}\right)$, $1708(\mathrm{C}=\mathrm{O}$ of $\mathrm{COOH}), 1617(\mathrm{C}=\mathrm{N}) ;{ }^{1} \mathrm{HNMR}\left(\mathrm{CDCl}_{3}+\mathrm{DMSO}-\mathrm{d}_{6}\right): \delta 12.14\left(\mathrm{OH}, \mathrm{s}, 1 \mathrm{H}, \mathrm{D}_{2} \mathrm{O}\right.$ exchangeable), 7.45-7.98( $\mathrm{Ar}-\mathrm{H}, \mathrm{m}, 9 \mathrm{H}), 3.22\left(\mathrm{CH}_{3}, \mathrm{~s}, 3 \mathrm{H}\right), 3.18\left(\mathrm{CH}_{3}, \mathrm{~s}, 3 \mathrm{H}\right), 2.63\left(\mathrm{CH}_{2}\right.$ $\left.\mathbf{C H}_{2} \mathrm{COOH}, \mathrm{t}, 2 \mathrm{H}\right) 2.39\left(\mathrm{CH}_{2} \mathrm{CH}_{2} \mathrm{COOH}, \mathrm{t}, 2 \mathrm{H}\right)$; MS: $\mathrm{m} / \mathrm{z}$ 321(M+1); Anal.Calcd for $\mathrm{C}_{20} \mathrm{H}_{20}$ $\mathrm{N}_{2} \mathrm{O}_{2}$ : C, 74.98; H, 6.29; N, 8.74.Found : C, 75.03; H, 6.26; N, $8.77 \%$.

4h: White solid: IR (KBr)cm ${ }^{-1}: 3423(\mathrm{O}-\mathrm{H}), 3014\left(\mathrm{C}-\mathrm{H}\right.$ of aromatic ring), 2930(C-H of $\left.\mathrm{CH}_{3}\right)$, $1710(\mathrm{C}=\mathrm{O}$ of $\mathrm{COOH}), 1623(\mathrm{C}=\mathrm{N}) ;{ }^{1} \mathrm{HNMR}\left(\mathrm{CDCl}_{3}+\mathrm{DMSO}^{\left.-\mathrm{d}_{6}\right)}: \delta 12.06\left(\mathrm{OH}, \mathrm{s}, 1 \mathrm{H}, \mathrm{D}_{2} \mathrm{O}\right.\right.$ exchangeable), 7.52-7.59( $\mathrm{Ar}-\mathrm{H}, \mathrm{m}, 9 \mathrm{H}), 3.08\left(\mathrm{OCH}_{3}, \mathrm{~s}, 3 \mathrm{H}\right), 2.64\left(\mathrm{CH}_{2} \mathrm{CH}_{2} \mathrm{COOH}, \mathrm{t}, 2 \mathrm{H}\right)$, 2.38 $\left(\mathrm{CH}_{2} \mathrm{CH}_{2} \mathrm{COOH}, \mathrm{t}, 2 \mathrm{H}\right)$; MS: $m / z$ 358(M+1); Anal.Calcd for $\mathrm{C}_{19} \mathrm{H}_{17} \mathrm{ClN}_{2} \mathrm{O}_{3}: \mathrm{C}, 63.96$; $\mathrm{H}, 4.80 ; \mathrm{N}, 7.85$. Found : C, 63.98; H, 4.78; N, 7.88\%.

4i: White solid: IR (KBr)cm ${ }^{-1}: 3426(\mathrm{O}-\mathrm{H}), 3012\left(\mathrm{C}-\mathrm{H}\right.$ of aromatic ring), 2932(C-H of $\mathrm{CH}_{3}$ ), $1718(\mathrm{C}=\mathrm{O}$ of $\mathrm{COOH}), 1619(\mathrm{C}=\mathrm{N}), 752(\mathrm{C}-\mathrm{Cl}) ;{ }^{1} \mathrm{HNMR}\left(\mathrm{CDCl}_{3}+\mathrm{DMSO}-\mathrm{d}_{6}\right): \delta 12.12(\mathrm{OH}$, s, $1 \mathrm{H}, \mathrm{D}_{2} \mathrm{O}$ exchangeable), 7.48-7.58( Ar- $\left.\mathrm{H}, \mathrm{m}, 9 \mathrm{H}\right), 3.04\left(\mathrm{CH}_{3}, \mathrm{~s}, 3 \mathrm{H}\right), 2.69\left(\mathrm{CH}_{2} \mathbf{C H}_{2} \mathrm{COOH}\right.$, t, $2 \mathrm{H}), 2.42\left(\mathrm{CH}_{2} \mathrm{CH}_{2} \mathrm{COOH}, \mathrm{t}, 2 \mathrm{H}\right)$; MS: $\mathrm{m} / z$ 342(M+1); Anal.Calcd for $\mathrm{C}_{19} \mathrm{H}_{17} \mathrm{ClN}_{2} \mathrm{O}_{2}: \mathrm{C}$, $66.96 ; \mathrm{H}, 5.03 ; \mathrm{N}, 8.22$. Found : C, 66.98; H, 5.00; N, 8.26\%.

4j: White solid: IR $(\mathrm{KBr}) \mathrm{cm}^{-1}: 3405(\mathrm{O}-\mathrm{H}), 3014(\mathrm{C}-\mathrm{H}$ of aromatic ring), 2815(C-H of $\left.\mathrm{CH}_{3}\right), 1708(\mathrm{C}=\mathrm{O}$ of $\mathrm{COOH}), 1617(\mathrm{C}=\mathrm{N}) ;{ }^{1} \mathrm{HNMR}\left(\mathrm{CDCl}_{3}+\mathrm{DMSO}-\mathrm{d}_{6}\right) ; \delta 12.11(\mathrm{OH}, \mathrm{s}, 1 \mathrm{H}$, 
$\mathrm{D}_{2} \mathrm{O}$ exchangeable), 7.45-7.98 (Ar-H, m,9H), 3.15( $\left.\mathrm{OCH}_{3}, \mathrm{~s}, 3 \mathrm{H}\right), 3.04\left(\mathrm{CH}_{3}, \mathrm{~s}, 3 \mathrm{H}\right), 2.63$ $\left(\mathrm{CH}_{2} \mathrm{CH}_{2} \mathrm{COOH}, \mathrm{t}, 2 \mathrm{H}\right), 2.40\left(\mathrm{CH}_{2} \mathrm{CH}_{2} \mathrm{COOH}\right)$; $\mathrm{MS}: \mathrm{m} / z$ 337(M+1);Anal.Calcd for $\mathrm{C}_{20} \mathrm{H}_{20} \mathrm{~N}_{2}$ $\mathrm{O}_{3}: \mathrm{C}, 71.41 ; \mathrm{H}, 5.99 ; \mathrm{N}, 8.33$.Found : C,71.43; H,5.97; $\mathrm{N}, 8.36 \%$.

\section{Conclusion}

The reduction of pyrazole acrylic acids to pyrazole propanoic acids using diimide method was found to have some advantages over palladium-charcoal method. The advantages are operational simplicity, reasonable yields, economical and easy work-up.

\section{Acknowledgment}

The authors are thankful to the management of G. Pulla Reddy College of Pharmacy, Hyderabad for providing facilities. The authors are also grateful to Director, IICT, Hyderabad and NACTO Pharma Limited, Hyderabad for providing spectral data.

\section{References}

1 Sahu S K, Banerjee M and Azam M A, Trop J Pharm Res., 2008, 7(2), 961-968.

2. Fabiane R S,Vanessa T S and Lysandro P B, Viviane R, Marli R O, Helio G B, Nilo Z, Marcos A P M and Carlos F M, Eur J Pharmacol., 2002, 451(2), 141-147.

3 Makhsumov A D,Kilichov G and Nikbaev A T, Pharm Chem J., 1986, 20, 289.

4 Bekhit A A, Ashour H M and Guemei A A, Arch Pharm(Weinheim), 2005, 338(4), 167-174.

5 İshak B, Ahmet S, Ekrem A and Abdulhamit B, Med Chem Res., 2009, 18(5), 327-340.

6 Brahmbhatt D I, Ankit R K, Anil K P annd Niraj H P, Indian J Chem., 2010, 49B, 971-977.

7 Guiping O, Xue-Jian C, Zhuo C, Bao-An S, Pinaki S. Bhadury, Song Yang, Lin-Hong Jin, Wei Xue, De-Yu Hu and Song Zeng, J Agric Food Chem., 2008, 56(21), 10160-10167.

8 Prakash O, Rashmi P, Pooja R, Kamaljeet P, Yogita D and Aneja K R, Indian $J$ Chem.., 2009, 48B, 563-568.

9 Bandgar B P, Totre J V, Gawande S S, Khobragade C N, Warangkar S C and Kadam P D, Bioorg Med Chem., 2010, 18(16), 6149-6155.

10 Nusrat B A and Rabiul M I, Bangladesh J Pharmacol., 2007, 2, 81.

11 Fotini N, Konstantinos M K, Prokopios M and Serkos A, Molecules, 2007, 12(7), 1259-1273.

12 Vilsmeier A and Haack A, Chem Ber., 1927, 60, 119.

13 Vogel's Text book of Practical Organic Chemistry, Vogel A I, Tatchell A R, Furnis B S, Hannaford A J and Smith P W G, Fifth Edition, 1191. 


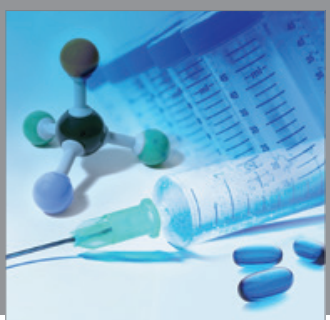

International Journal of

Medicinal Chemistry

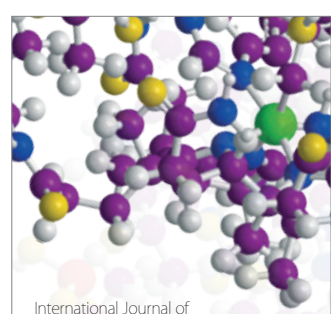

Carbohydrate Chemistry

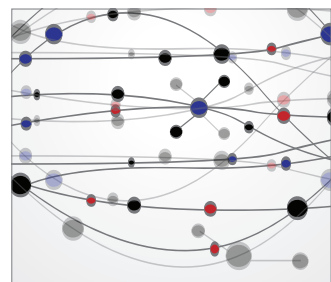

The Scientific World Journal
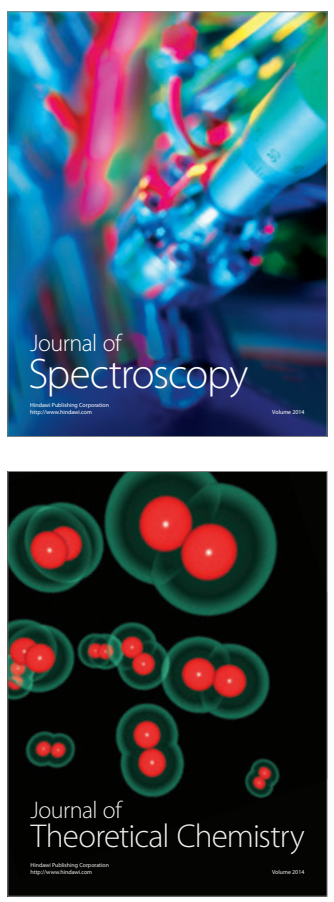
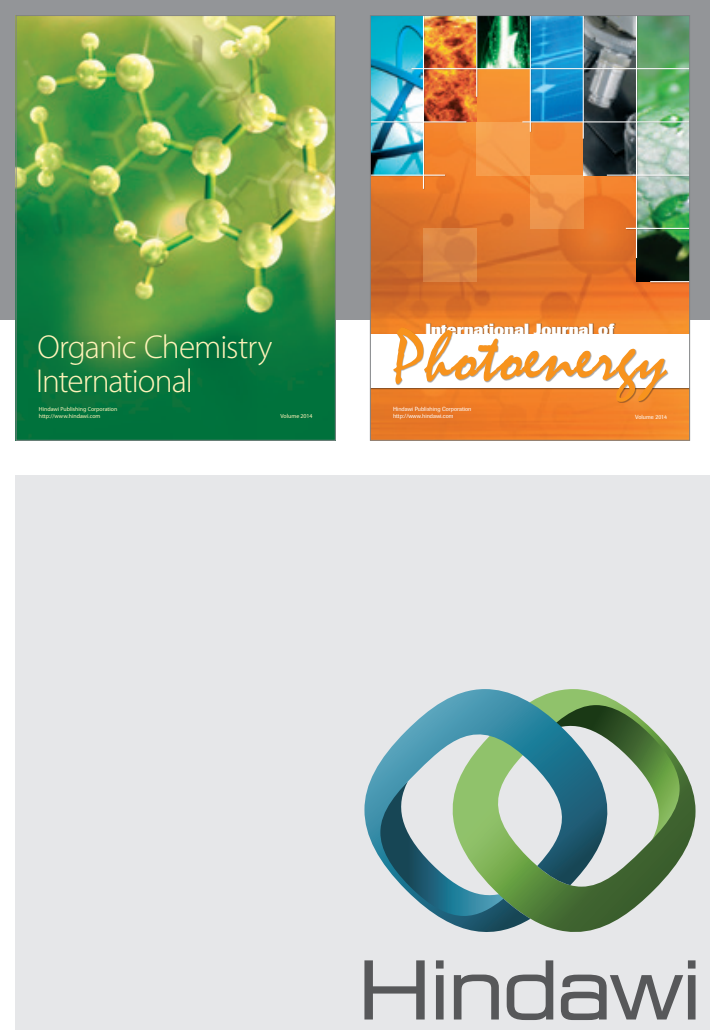

Submit your manuscripts at

http://www.hindawi.com
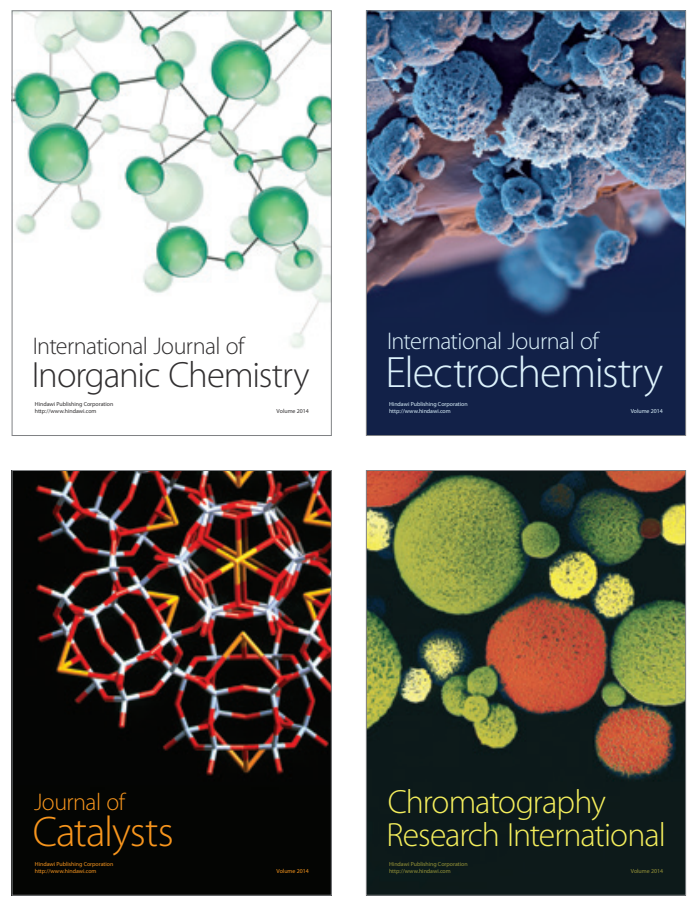
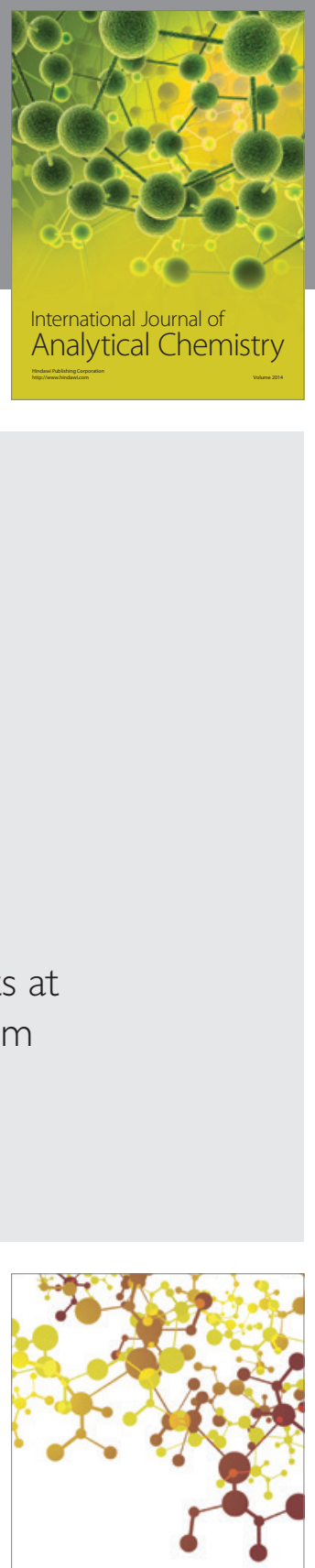

Journal of

Applied Chemistry
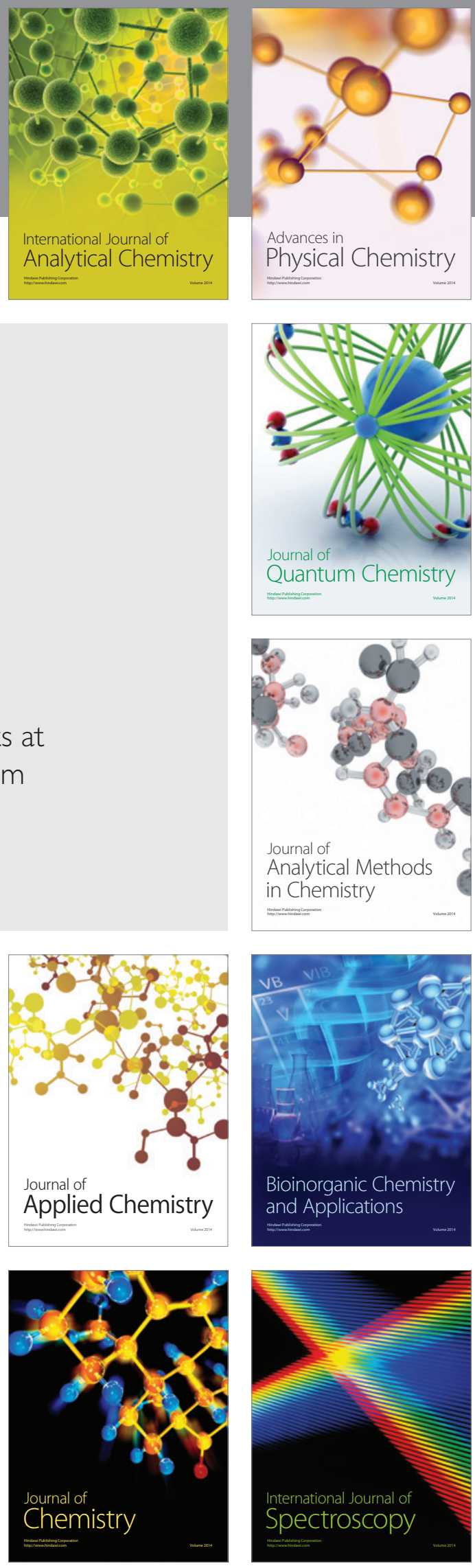